\title{
Modulation of Prrxl1 transcriptional activity by phosphorylation
}

\author{
AS Pessoa ${ }^{2}$, R Soares-dos-Reis ${ }^{2}$, M Falcão ${ }^{2}$, M Matos $^{2}$, CB Monteiro ${ }^{2}$, FA Monteiro ${ }^{2}$, C Reguenga ${ }^{2 *}$, D Lima ${ }^{1,2}$ \\ From International Conference for Healthcare and Medical Students 2011 \\ Dublin, Ireland. 4-5 November 2011
}

\section{Introduction}

Prrxl1 is a homeodomain transcription factor essential for the connectivity and survival of nociceptive neurons in the mouse embryo dorsal root ganglion (DRG) and spinal cord (SC). Prrxl1 $1^{-1-}$ mice show altered patterning of nociceptive afferent projections to the dorsal SC (dSC), neuronal loss, reduced nociception and failure to thrive. Prrxl1 displays a multiple band pattern on western-blots (WB), which is abrogated by incubation with a phosphatase. Our aim is to further characterize the nature and sites of Prrxl1 post-translational modifications and their impact on its function.

\section{Methods}

Mice were dissected and nuclear extracts of DRG and dSC of mouse embryos (from embryonic day 12.5 to post-natal day 14) were prepared using a Triton-sucrose buffer and analysed by WB. This time-course analysis was further completed by 2D-electrophoresis (isoelectric focusing followed by SDS-PAGE) of embryonic and post-natal dSC extracts. Furthermore, nuclei of ND7/23 cells (a DRGderived cell-line with nociceptive properties) and embryonic $\mathrm{dSC}$ were incubated with a $\mathrm{GA}^{3+}$ immobilized metal ion affinity chromatography resin, with affinity for phosphopeptides. Constructs corresponding to truncated versions of Prrxl1 were generated by molecular cloning, and their WB band pattern, transcriptional activity and DNAbinding activity were assessed by luciferase-reporter assays and by a modified DNA pull-down assay. $\mathrm{CNBr}$ chemical cleavage of recombinant protein was performed to enhance this analysis. Moreover, site-directed mutagenesis was performed for an evolutionarily conserved putative

\footnotetext{
* Correspondence: cregueng@med.up.pt

${ }^{2}$ IBMC Instituto de Biologia Molecular e Celular, Universidade do Porto,

Portugal

Full list of author information is available at the end of the article
}

phosphorylation site which was subjected to the same analysis.

\section{Results}

Prrxl1 is highly phosphorylated and its phosphorylation state varies in a time and tissue specific manner. Phosphorylation seems to occur throughout the protein, but mainly in the N-terminus (encompassing a DNA-binding domain) and in the C-terminus (containing a conserved putative regulatory domain). Evolutionary conserved site analysis revealed a highly conserved phosphorylation site in the homedomain, whose mutation impairs transcriptional activation, but not DNA-binding. Luciferase reporter assays of truncated versions of Prrxl1 have identified the $\mathrm{N}$-terminus as sufficient for the DNA-binding and transcriptional activity of Prrxll.

\section{Conclusions}

Altogether, our results show that Prrxl1 is mainly phosphorylated in the $\mathrm{C}$-terminus and in the $\mathrm{N}$-terminus. Moreover, these phosphorylations probably play a fundamental role in the regulation of Prrxl1 transcriptional activity in nociceptive neurons.

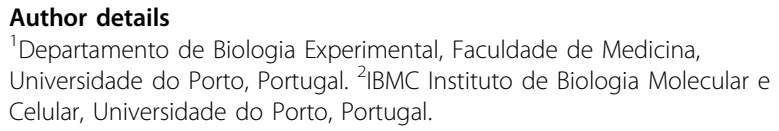

Published: 9 July 2012

doi:10.1186/1753-6561-6-S4-047

Cite this article as: Pessoa et al:: Modulation of Prrxl1 transcriptional activity by phosphorylation. BMC Proceedings 2012 6(Suppl 4):O47.
C 2012 Pessoa et al; licensee BioMed Central Ltd. This is an Open Access article distributed under the terms of the Creative Commons Attribution License (http://creativecommons.org/licenses/by/2.0), which permits unrestricted use, distribution, and reproduction in any medium, provided the original work is properly cited. 